\title{
Colonização pré-operatória por Staphylococcus aureus e as bactérias não suscetíveis à cefalosporina, em pacientes com fratura proximal do fêmur*
}

\section{Pre-operative Colonization by Staphylococcus aureus and Cephalosporin Non-susceptible Bacteria in Patients with Proximal Femoral Fractures}

\author{
Leonardo R. Bastos ${ }^{1,2(0)}$ Mila M. Almeida ${ }^{20}$ Elizabeth A. Marques ${ }^{20}$ Robson Souza Leão $2,3(0)$ \\ 1 Seção de Ortopedia e Traumatologia, Hospital Geral de \\ Fortaleza/Exército Brasileiro, Fortaleza, CE, Brasil \\ 2 Departamento de Microbiologia, Imunologia e Parasitologia, \\ Faculdade de Ciências Médicas, Universidade do Estado do Rio de \\ Janeiro, RJ, Brasil

\begin{abstract}
Endereço para correspondência Robson Souza Leão, PhD, Departamento de Microbiologia, Imunologia e Parasitologia, Faculdade de Ciências Médicas, Universidade do Estado do Rio de Janeiro, Avenida 28 de Setembro, S/N, Vila Isabel, Rio de Janeiro, RJ, Brasil (e-mail: robson.leao@uerj.br).
\end{abstract}

3 Laboratórios de Bacteriologia e Micobactérias, Hospital Universitário Pedro Ernesto, Universidade do Estado do Rio de Janeiro, Rio de Janeiro, RJ, Brasil

Rev Bras Ortop 2022;57(5):726-733.

\section{Resumo \\ Palavras-chave \\ - portador sadio \\ - resistência a medicamentos \\ - fraturas do fêmur \\ - Enterobacteriaceae \\ - fatores de risco \\ - Staphylococcus}

Objetivo Estimar a frequência da colonização por Staphylococcus aureus e as bactérias não suscetíveis à cefalosporina, em pacientes com fratura proximal do fêmur durante a internação pré-operatória.

Métodos Avaliação da prevalência e incidência em 63 pacientes hospitalizados ao longo de um ano. O tempo médio de internação pré-tratamento foi de 12 dias. As amostras foram coletadas das narinas, pele da virilha e mucosa anal, durante a internação prévia ao tratamento e testadas pela técnica de disco-difusão.

Resultados A incidência da colonização hospitalar e a prevalência de resultados positivos foram de $14,3 \%$ e $44,4 \%$ para Staphylococcus aureus; $3,2 \%$ e $6,4 \%$ para S. aureus resistente à meticilina; $28,6 \%$ e $85,7 \%$ para Staphylococcus coagulase-negativo resistente à meticilina; $28,6 \%$ e $61,9 \%$ para Enterobacteriaceae não suscetível à cefazolina (KFNSE); e 20,6\% e 28,6\% para Enterobacteriaceae não suscetível à cefuroxima (CXNSE).

\footnotetext{
Trabalho desenvolvido no Departamento de Microbiologia, Imunologia e Parasitologia, Faculdade de Ciências Médicas, Universidade do Estado do Rio de Janeiro e do Hospital Geral de Fortaleza/Exército Brasileiro, Fortaleza, Ceará, Brasil
}

recebido

10 de Dezembro de 2020

aceito

07 de Abril de 2021

Publicado on-line

de Janeiro 5, 2022
DOI https://doi.org/ 10.1055/s-0041-1735546. ISSN 0102-3616.
(C) 2022. Sociedade Brasileira de Ortopedia e Traumatologia. All rights reserved.

This is an open access article published by Thieme under the terms of the Creative Commons Attribution-NonDerivative-NonCommercial-License, permitting copying and reproduction so long as the original work is given appropriate credit. Contents may not be used for commercial purposes, or adapted, remixed, transformed or built upon. (https://creativecommons.org/ licenses/by-nc-nd/4.0/)

Thieme Revinter Publicações Ltda., Rua do Matoso 170, Rio de Janeiro, RJ, CEP 20270-135, Brazil 


\begin{abstract}
Keywords

- carrier state

- drug resistance

- Enterobacteriaceae

- femoral fractures

- risk factors

- Staphylococcus

Objective To estimate the frequency of Staphylococcus aureus and cephalosporin nonsusceptible bacteria colonization in patients with proximal femoral fracture during preoperative hospitalization.

Methods Prevalence and incidence assessment in 63 hospitalized patients over 1 year. The median time of pretreatment hospitalization was 12 days. Samples were collected from the nostrils, groin skin and anal mucosa during the pretreatment hospitalization and were tested by the disc-diffusion technique.

Results The hospital colonization incidence and the prevalence of positive results were 14.3 and $44.4 \%$ for S. aureus; 3.2 and $6.4 \%$ for meticillin-resistant S. aureus; 28.6 and $85.7 \%$ for meticillin-resistant coagulase-negative Staphylococcus; 28.6 and 61.9\% for cefazolin nonsusceptible Enterobacteriaceae (KFNSE); and 20.6 and 28.6\% for cefuroxime nonsusceptible Enterobacteriaceae (CXNSE). In addition, factors such as to the duration of the pretreatment hospitalization period, being non-walker before fracture, antimicrobial use, American Society of Anesthesiologists (ASA) 4 surgical risk, and previous hospitalization, were related to an increase in the incidence of hospital acquisition and prevalence of colonization by the evaluated strains. The prevalence of colonization by KFNSE was three times higher than by CXNSE on admission, and twice as high at the time of fracture treatment.

Conclusion There was a high incidence of hospital colonization and prevalence of colonization by all strains studied, which may guide the indication of prophylactic measures for infection.
\end{abstract}

Além da duração do período de internação pré-tratamento, os pacientes não deambularam previamente à ocorrência da fratura e nem fizeram uso de antimicrobiano. Além disso, a duração do período de internação pré-tratamento cirúrgico, ser nãodeambulador antes da fratura, uso de antimicrobianos, risco cirúrgico IV pela American Society of Anesthesiologists (ASA) e internação anterior, estiveram relacionados a um aumento na incidência de aquisição hospitalar e prevalência de colonização pelas cepas avaliadas. A prevalência de colonização pela KFNSE foi três vezes maior do que pela CXNSE na admissão e duas vezes maior no momento do tratamento da fratura.

Conclusão Observou-se uma alta incidência da colonização hospitalar e prevalência da colonização por todas as cepas estudadas, o que pode orientar a indicação de medidas profiláticas contra a infecção.

\section{Introdução}

Nos países de baixa renda, a causa mais comum das infecções associadas ao tratamento de saúde, são as infecções do sítio cirúrgico (ISC), incluindo os procedimentos ortopédicos. $^{1}$ O Staphylococcus aureus é o principal agente etiológico após fraturas proximal do fêmur (FPF), seguido por Staphylococcus coagulase-negativo (CoNS) e Enterobacteriaceae. $^{2}$

É evidente a importância da colonização por S. aureus na gênese da ISC, já que é o principal fator de risco. Uma das medidas propostas para prevenir as ISC é a descolonização dos pacientes. ${ }^{1,3}$

A profilaxia antibiótica pré-operatória é uma medida comprovada para a prevenção da ISC em pacientes com $\mathrm{FPF}^{4}$ As cefalosporinas de ação prolongada são as mais indicadas, não havendo consenso quanto ao uso de cefazolina ou cefuroxima. ${ }^{5}$
No entanto, a eficácia está relacionada à suscetibilidade, podendo ser necessário o ajuste do tratamento. ${ }^{6}$

As infecções causadas por $S$. aureus resistente à meticilina (SARM) tornam ineficaz o tratamento com $\beta$-lactâmicos, já tendo sido descrita sua disseminação em ambientes não hospitalares colonizando indivíduos saudáveis. ${ }^{7}$ A alta incidência de Staphylococcus coagulase-negativos resistentes à meticilina (SCoNRM), ${ }^{8}$ pode comprometer a eficácia das cefalosporinas como antibiótico profilático. Da mesma forma, as cefalosporinas são incapazes de prevenir a disseminação das Enterobacteriaceae que infectam o sítio cirúrgico e produzem $\beta$-lactamases. ${ }^{9,10}$

O objetivo deste estudo foi estimar a frequência da colonização por Staphylococcus e Enterobacteriaceae envolvidos na ISC e não suscetíveis aos antibióticos comumente usados na profilaxia intraoperatória em pacientes com FPF, bem como avaliar o impacto da hospitalização pré-operatória prolongada e outros fatores de risco. 


\section{Métodos}

Foram avaliados todos os pacientes internados consecutivamente entre abril de 2015 e março de 2016, em um hospital militar do Rio de Janeiro para tratamento de FPF. Os critérios de inclusão foram que as fraturas fossem causadas por traumas de baixa energia. Os critérios de exclusão foram pacientes hospitalizados para tratamento de complicações de uma fratura do fêmur tratada anteriormente. Dos 66 pacientes internados que atenderam aos critérios de inclusão, três foram excluídos por não concordarem em participar.

As amostras para triagem foram coletadas com um swab na região anterior das narinas, na pele da virilha no lado da fratura e na mucosa anal, sendo semeadas em Sal Manitol e Agar MacConkey, respectivamente. As coletas foram realizadas no momento da admissão (até 72h - amostra 1), entre $72 \mathrm{~h}$ e sete dias de internação (amostra 2 ), e uma vez por semana após a primeira semana (amostras 3 a 9), até a data da osteossíntese femoral, a definição por tratamento não cirúrgico ou a ocorrência do óbito antes do tratamento.

A identificação bacteriana foi realizada por meio do espectrômetro de massa Microflex/Bruker - ionização e dessorção à laser assistida por matriz e analisador de tempo-de-voo.

A susceptibilidade aos antibióticos foi obtida por discodifusão, de acordo com o Instituto Norte-americano de Padrões Clínicos e Laboratoriais (CLSI). ${ }^{11}$ A Cefoxitina foi realizada para CoNS e para SARM, sendo também avaliados os fármacos ciprofloxacina, eritromicina, clindamicina, sulfametoxazol/trimetoprima, linezolida e rifampicina. Quanto as Enterobacteriaceae, foram empregados os fármacos cefazolina e cefuroxima. Os resultados intermediários e resistentes foram classificados como "não suscetíveis".
Todos os isolados de SARM foram submetidos à análise de reação em cadeia da polimerase (PCR) para tipagem dos $\mathrm{SCCmec},^{12}$ genes lukS-PV relacionados à produção de leucocidina Panton-Valentine (PVL) ${ }^{13}$ e os genes $q a c A / B,{ }^{14}$ relacionados à resistência à clorexidina.

Após o primeiro resultado positivo para bactérias não suscetíveis, os pacientes foram considerados colonizados. A colonização dos pacientes cuja triagem de admissão foi negativa para bactérias resistentes à antibióticos foi classificada como colonização hospitalar. A análise da incidência da colonização hospitalar foi realizada a partir da segunda amostra, considerando os pacientes com resultados negativos em todas as amostras anteriores.

Os fatores de risco para a colonização por bactérias resistentes a antibióticos, foram adaptados dos fatores de risco para colonização por SARM ${ }^{15}$ (-Tabela 1). A análise estatística da relação entre a incidência da colonização hospitalar e o tempo médio de internação antes do inicio do tratamento da fratura ou o óbito antes do tratamento, foi realizada com o teste de Mann-Whitney para as duas amostras. Foram realizadas usando o teste exato de Fisher, a análise estatística da relação entre o risco de colonização por bactérias não suscetíveis à antibióticos, a incidência de colonização hospitalar, a prevalência da colonização na triagem de admissão ou em qualquer amostra antes do tratamento da fratura do fêmur, além da incidência de cefazolina ou cefuroxima não suscetíveis às Enterobacteriaceae. Em ambos os casos, Foi rejeitada a hipótese nula para valores de $p>0,05$. Todas as análises estatísticas foram realizadas com a utilização do programa Epi Info TM 7.1.5.2.

Todos os pacientes foram acompanhados por um período mínimo de um ano (exceto em caso de óbito), em consultas ambulatoriais ou em contatos telefônicos.

Tabela 1 Prevalência dos fatores de risco para a colonização por bactérias não suscetíveis aos antibióticos, na população estudada no momento da internação (admissão hospitalar) e no tratamento da fratura femoral ou óbito anterior ao tratamento, (data do tratamento)

\begin{tabular}{|c|c|c|}
\hline & Internação hospitalar & Data do tratamento \\
\hline \multicolumn{3}{|l|}{ Fator de Risco } \\
\hline Acamados $^{\mathrm{a}}$ & $11(17,5 \%)$ & $11(17,5 \%)$ \\
\hline Diabetes mellitus & $19(30,2 \%)$ & $19(30,2 \%)$ \\
\hline Hospitalização prévia à ocorrência da fratura (últimos 12 meses) & $13(20,6 \%)$ & $13(20,6 \%)$ \\
\hline Uso de antibióticos ${ }^{\mathrm{b}}$ & $20(31,7 \%)$ & $37(58,73 \%)$ \\
\hline Institucionalizados ${ }^{c}$ & $13(20,6 \%)$ & $13(20,6 \%)$ \\
\hline Úlceras de pressão ${ }^{d}$ & $7(11,1 \%)$ & $13(20,6 \%)$ \\
\hline ASA IV & $20(31,75 \%)$ & $20(31,75 \%)$ \\
\hline Cateterismo vesical $^{f}$ & 0 & $13(20,63 \%)$ \\
\hline Permanência na UTI ${ }^{f}$ & 0 & $8(12,7 \%)$ \\
\hline
\end{tabular}

aPacientes que se moviam apenas com o auxílio de uma cadeira de rodas ou que estavam acamados antes da ocorrência da fratura

bUso de antibióticos antes da hospitalização (6 meses), ou durante a hospitalização e antes do tratamento da fratura

'Pacientes hospitalizados ou residentes em casa de repouso, hospitalização domiciliar ou sob tratamento de hemodiálise

dPresença de úlceras de pressão na admissão hospitalar ou adquiridas durante a internação e antes do tratamento da fratura

eASA, classificação do estado físico da American Society of Anesthesiologists (16)

${ }^{\mathrm{f}}$ Cateterismo vesical ou permanência na UTI, ocorrido durante a hospitalização e antes da fratura 


\section{Aspectos Éticos}

O protocolo da pesquisa foi aprovado por um comitê de ética local e todos os pacientes incluídos ou seus responsáveis legais, assinaram o termo de consentimento livre e esclarecido (CAAE: 39070314.0.0000.5256). Os dados clínicos foram obtidos por meio de entrevistas e prontuários médico-hospitalar.

\section{Resultados}

A idade média dos pacientes foi $79 \pm 10$ anos e $79,4 \%$ eram do sexo feminino. A fratura trocantérica ocorreu em 28 (44,4\%) pacientes, 26 (41,3\%) apresentaram fratura do colo femoral, sete $(11,1 \%)$ subtrocantérica e dois pacientes $(3,2 \%)$ que tiveram fratura isolada do trocânter maior. Na - Tabela 1 está demonstrada a prevalência dos fatores de risco para a colonização por bactérias não suscetíveis aos antibióticos na população estudada, no momento da admissão hospitalar e na data do tratamento da fratura,.

O tratamento da fratura foi realizado com fixação em 22 (34,9\%) pacientes, com parafuso dinâmico de quadril e placa 10 $(15,9 \%)$ pacientes, artroplastia parcial do quadril em outros 10 pacientes (15,9\%), artroplastia total do quadril em sete (11,1\%) pacientes, dois casos $(3,2 \%)$ com parafuso condilar dinâmico e placa e um caso $(1,6 \%)$ tratado com artroplastia de ressecção. A cefazolina foi aplicada durante a indução anestésica em todos os casos cirúrgicos. O tempo médio cirúrgico foi de $103,4 \pm 39,5$ minutos. Cinco pacientes (7,9\%) não foram submetidos ao tratamento cirúrgico, em virtude do alto risco cirúrgico e condições clínicas desfavoráveis, dois $(3,2 \%)$ devido ao padrão da fratura e quatro pacientes (6,3\%) faleceram antes da possibilidade de tratamento cirúrgico.

O tempo médio de internação prévia ao tratamento da fratura ou o óbito antes do tratamento foi de 12 dias ( $I Q R=8-$ 19). Dos 63 pacientes avaliados, apenas um recebeu tratamento para a fratura em menos de três dias, 15 receberam tratamento entre três e sete dias, 24 entre oito e quatorze dias e 23 após quatorze dias de internação.

A amostra 1 foi coletada em todos os pacientes. A amostra 2 foi coletada em 62 pacientes, amostra 3 em 50, amostra 4 em 22 pacientes, amostra 5 em oito pacientes, amostra 6 em cinco pacientes, amostra 7 em dois pacientes e amostra 8 e 9 em um paciente.

Na região nasal e na virilha, foram identificados 637 isolados de Staphylococcus, bem como 377 isolados de Enterobacteriaceae obtidos nas amostras anais. A prevalência de resultados positivos nas amostras de triagem da admissão foi de $30,2 \%$ para S. aureus, 3,2\% para SARM; 57,1\% para SCoNRM; 33,3\% para Enterobacteriaceae não sensível à cefazolina (KFNSE); e 7,9\% para Enterobacteriaceae não sensível à cefuroxima (CXNSE).

A incidência de colonização hospitalar e a prevalência dos resultados positivos em qualquer amostra antes do tratamento ou óbito antes do tratamento, foram de $14,3 \%$ e $44,4 \%$ para $S$. aureus; $3,2 \%$ e $6,4 \%$ para SARM; $28,6 \%$ e $85,7 \%$ para SCoNRM; $28,6 \%$ e $61,9 \%$ para KFNSE; e 20,6\% e 28,6\% para CXNSE.

Durante o período de acompanhamento, foram diagnosticadas três infecções do sítio cirúrgico, uma causada por SARM, outra por Enterobacteriaceae e outra por SCONRM (S. epidermidis e $S$. haemolyticus).

\section{Staphylococcus aureus}

Foram identificados 89 isolados de S. aureus, sendo a narina (77,5\%) o local de identificação mais frequente. Houve um aumento progressivo na incidência de colonização hospitalar por S. aureus (-Fig. 1). No período entre 14 e 21 dias de internação, $18 \%$ dos pacientes que ainda estavam internados apresentaram colonização. Nesse mesmo período, 50\% dos pacientes que permaneceram internados apresentaram alguma amostra positiva para S. aureus (-Fig. 1).

Analisando o grupo de pacientes com resultados negativos para S. aureus na triagem de admissão (44 pacientes), observamos que a mediana do tempo de internação prévio ao tratamento da fratura ou óbito antes do tratamento, nos pacientes que adquiriram colonização hospitalar foi de 17 dias $(\mathrm{IQR}=15,31)$, contra 11 dias $(\mathrm{IQR}=9,18)$ naqueles que não adquiriram $(p=0,06)$.

Dois pacientes apresentaram resultado positivo no rastreamento da admissão (3,2\%), apenas no swab da virilha. Um deles apresentou infecção pós-operatória superficial causada por SARM, diagnosticada por cultura de secreção obtida por meio de punção do sítio cirúrgico e tratada com antibióticos.

Dois outros pacientes apresentaram amostras positivas para SARM, com triagem de admissão negativa. Todos os pacientes testados para SARM, foram positivos para SCCmec$I V$, negativos para os genes lukS-PV e qacA/B e suscetíveis a sulfametoxazol/trimetoprima.

\section{Estafilococos Coagulase-Negativos}

Cerca de $43 \%$ dos SCoN foram identificados apenas até o nível de gênero. O S. haemolyticus (29,6\%) e o S. epidermidis (20,6\%) foram os mais observados. $O$ mais isolado foi o $S$. haemolyticus $(43,2 \%)$ no SCoNRM.

$\mathrm{O}$ auge da incidência da colonização hospitalar por SCoNRM ocorreu entre o $4^{\circ}$ e o $7^{\circ}$ dia de internação, atingindo $21 \%$ dos pacientes internados naquele momento, com prevalência de $77,4 \%$ dos pacientes já colonizados por SCONRM (-Fig. 1).

Analisando os pacientes com resultados negativos para SCoNRM na triagem para internação (27 pacientes), observamos que o tempo médio de internação antes do tratamento da fratura ou óbito antes do tratamento, em pacientes que adquiriram colonização hospitalar foi de 17 dias ( $I Q R=12,22)$, contra 9 dias $(\mathrm{IQR}=8,12)$ naqueles que não adquiriram $(p<0,01)$.

Um paciente com duas amostras de rastreamento positivas $\left(1^{\mathrm{a}}\right.$ e $\left.2^{\mathrm{a}}\right)$ para SCoNRM, apresentou osteomielite pósoperatória por S. haemolyticus e S. epidermidis, diagnosticada por cultura de fragmento ósseo, obtida durante a retirada da prótese total do quadril.

\section{Enterobacteriaceae}

Das 377 Enterobacteriaceae, 100 (26,5\%) apresentaram resultado não sensível à cefazolina (KFNSE) e 30 (8\%) à cefuroxima (CXNSE) $(p<0,01)$. A Escherichia coli $(62,1 \%)$ foi a mais isolada, seguida pela Proteus mirabilis (17,2\%) e Klebsiella pneumoniae $(5,8 \%)$.

$O$ auge da incidência da colonização hospitalar por KFNSE e CXNSE ocorreu entre o $4^{\circ}$ e o $7^{\circ}$ dia de internação, após o qual $50 \%$ dos pacientes já estavam colonizados por KFNSE e 18\% por CXNSE (-Fig. 1 ). 

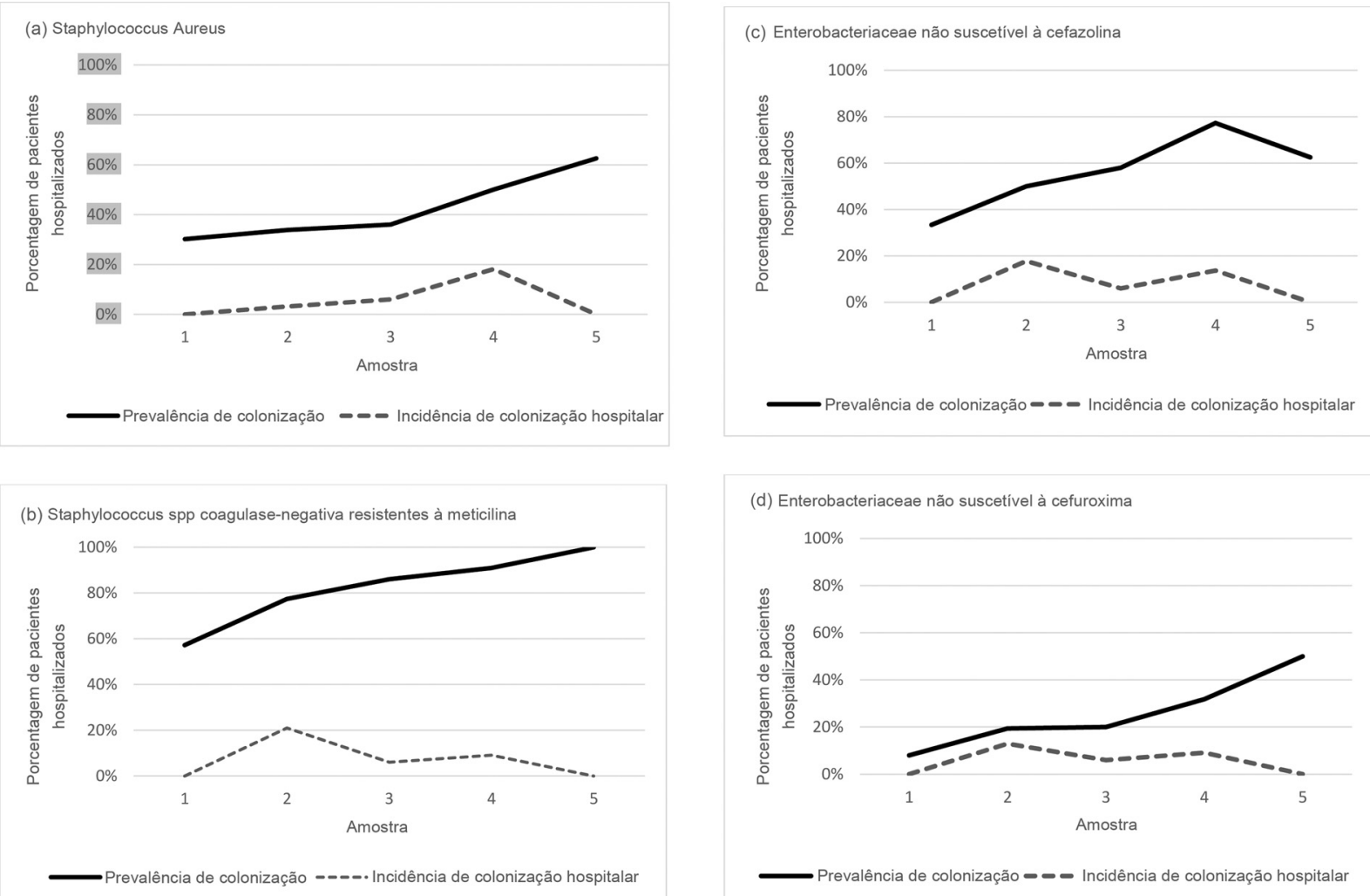

Fig. 1 Prevalência de pacientes colonizados e a incidência de colonização hospitalar para cada grupo de bactérias estudadas, desde a amostra de triagem na admissão (1 ${ }^{\text {a }}$ ) até a quinta amostra de triagem

O tempo médio de internação hospitalar antes do tratamento da fratura ou óbito antes do tratamento, nos pacientes que adquiriram colonização hospitalar por KFNSE foi de 17 dias $(\mathrm{IQR}=14,25)$, contra 9 dias $(\mathrm{IQR}=7,12)$ naqueles que não adquiriram $(p<0,01)$. Nos pacientes que adquiriram a colonização hospitalar por CXNSE, foi de 17 dias ( IQR $=10,22)$, contra 12 dias $(\mathrm{IQR}=9,17)$ naqueles que não adquiriram $(p=0,34)$.

Um paciente apresentou infecção pós-operatória por Enterobacteriaceae intrinsecamente resistente às cefalosporinas, diagnosticada por meio de cultura de fragmentos ósseos, obtidos durante o desbridamento cirúrgico e confirmada por outra cultura de fragmento ósseo, obtida durante a retirada da hemiartroplastia. As três amostras de rastreamento pré-operatório desse paciente foram negativas para KFNSE ou CXNSE.

\section{Fatores de Risco}

São mostradas nas - Tabelas $\mathbf{2}$ e $\mathbf{3}$ a razão de prevalência da colonização na admissão ou em qualquer triagem antes do tratamento da fratura ou óbito antes do tratamento e a proporção de risco da colonização hospitalar, de acordo com a presença de um dos fatores de risco para a colonização por bactérias não suscetíveis aos antibióticos.

\section{Discussão}

O hospital no qual foi realizado o presente estudo enfrentou várias dificuldades, em relação ao grande número de pacientes internados, à disponibilidade de salas cirúrgicas e de equipe médica, aumentando o tempo de internação antes do tratamento da fratura femoral. Embora essas não sejam as condições ideais para o tratamento de pacientes com FPF, essa situação é comum nos hospitais públicos brasileiros. Assim, a análise desses dados pode permitir a montagem de estratégias para minimizar as infecções pós-operatórias em situações semelhantes.

Aqui, $30 \%$ dos pacientes apresentavam S. aureus na triagem de admissão, semelhante aos dados da literatura. ${ }^{16}$ No período de internação prévio ao tratamento da fratura, outros $14 \%$ dos pacientes foram colonizados por S. aureus. Considerando a importância da colonização pré-operatória nas ISC, ${ }^{1}$ o alto índice observado de colonização hospitalar sugere a necessidade de implantação de protocolos de controle, principalmente em pacientes com fatores de risco relacionados, que em nossa série foram os que não deambulavam, faziam uso de antimicrobianos, institucionalizados e internados em UTI.

O número reduzido de pacientes que apresentaram colonização por SARM não permite analisar os fatores de risco ou os padrões de disseminação. No entanto, destacamos que todos os pacientes que apresentaram colonização por SARM, eram SCCmec tipo IV e suscetíveis à sulfametoxazol/trimetoprima, sugerindo cepas de SARM adquirido na comunidade (CA-MRSA). ${ }^{17}$ Além disso, a predominância de positivos nas amostras da virilha mostram a importância do rastreamento do SARM em múltiplos sítios. ${ }^{18}$ Todavia, ainda está em discussão a relação entre a colonização não nasal e não mucosa, além do aumento da taxa da ISC. ${ }^{19,20}$ 


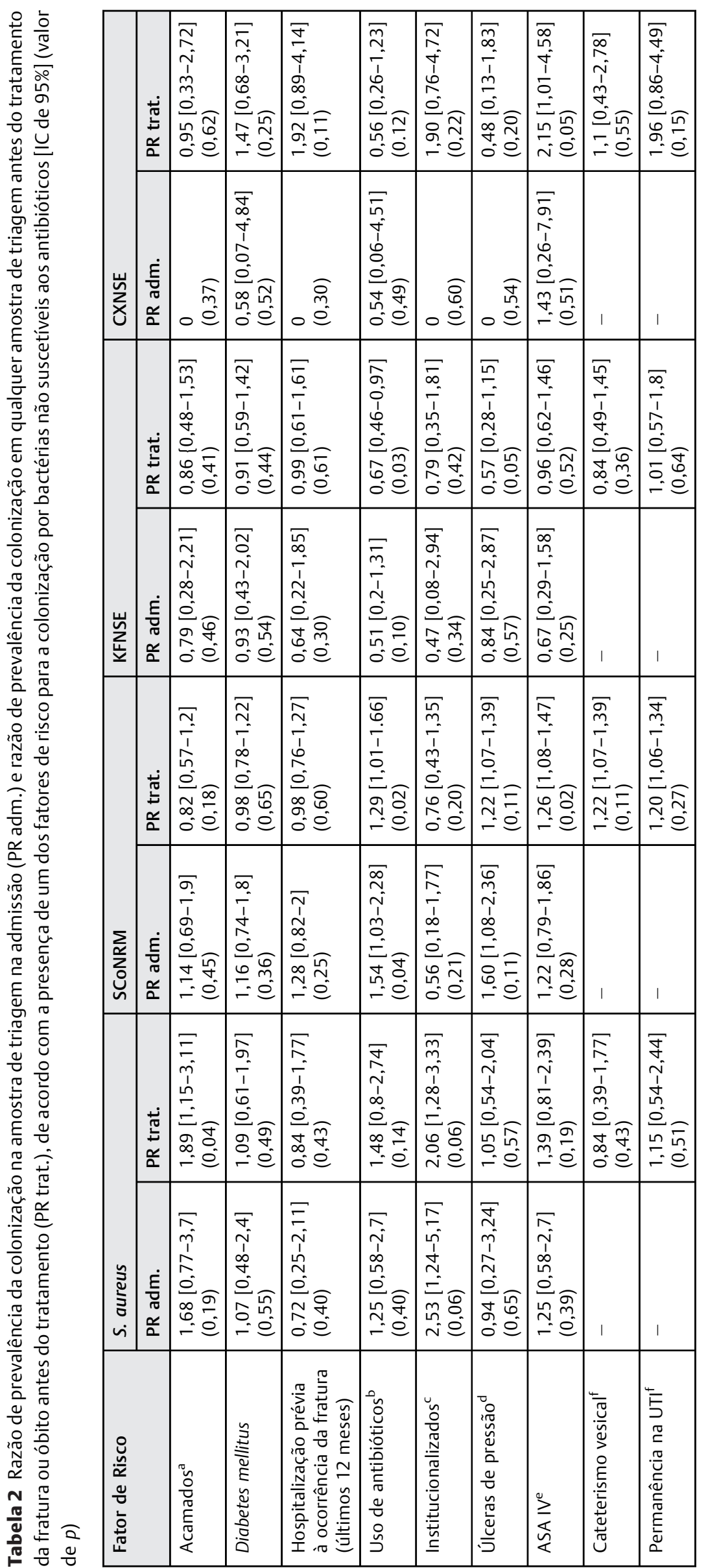

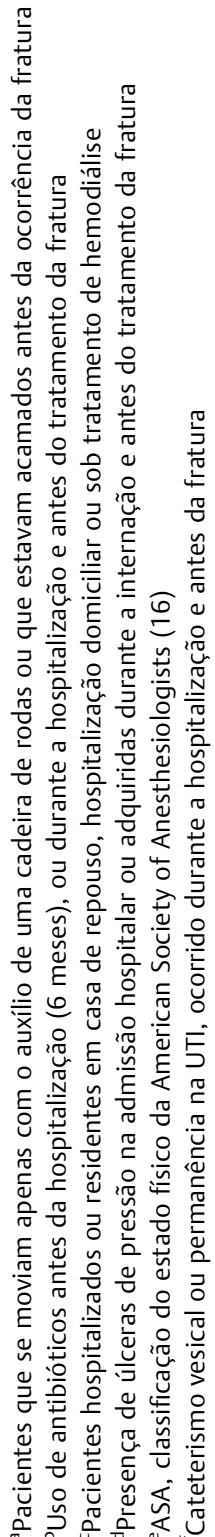


Tabela 3 Razão de risco da colonização hospitalar (RR ch), de acordo com a presença de um dos fatores de risco para colonização por bactérias não suscetíveis aos antibióticos [IC de 95\%] (valor de $p$ )

\begin{tabular}{|c|c|c|c|c|}
\hline Fator de Risco & S. aureus & SCONRM & KFNSE & CXNSE \\
\hline acamados $^{\mathrm{a}}$ & $\begin{array}{l}3,17[1,07-9,38] \\
(0,09)\end{array}$ & $\begin{array}{l}0,34[0,06-1,88] \\
(0,09)\end{array}$ & $\begin{array}{l}0,85[0,32-2,25] \\
(0,53)\end{array}$ & $\begin{array}{l}1,28[0,42-3,89] \\
(0,47)\end{array}$ \\
\hline Diabetes mellitus & $\begin{array}{l}1,19[0,35-4,06] \\
(0,54)\end{array}$ & $\begin{array}{l}0,82[0,40-1,65] \\
(0,43)\end{array}$ & $\begin{array}{l}0,86[0,39-1,90] \\
(0,48)\end{array}$ & $\begin{array}{l}1,91[0,75-4,87] \\
(0,15)\end{array}$ \\
\hline $\begin{array}{l}\text { Hospitalização prévia à } \\
\text { ocorrência da fratura } \\
\text { (últimos } 12 \text { meses) }\end{array}$ & $\begin{array}{l}0,97[0,24-3,96] \\
(0,67)\end{array}$ & $\begin{array}{l}0,72[0,26-1,99 \\
(0,41)\end{array}$ & $\begin{array}{l}1,23[0,58-2,6] \\
(0,43)\end{array}$ & $\begin{array}{l}2,97[1,21-7,29] \\
(0,03)\end{array}$ \\
\hline Uso de antibióticos ${ }^{\mathrm{b}}$ & $\begin{array}{l}5,54[0,76-40,52] \\
(0,04)\end{array}$ & $\begin{array}{l}1,56[0,91-2,67] \\
(0,11)\end{array}$ & $\begin{array}{l}0,62[0,32-1,22] \\
(0,16)\end{array}$ & $\begin{array}{l}0,56[0,22-1,46] \\
(0,19)\end{array}$ \\
\hline Institucionalizados ${ }^{c}$ & $\begin{array}{l}2,62[0,57-12] \\
(0,37)\end{array}$ & $\begin{array}{l}0,72[0,26-1,99] \\
(0,41)\end{array}$ & $\begin{array}{l}0,92[0,3-2,88] \\
(0,64)\end{array}$ & $\begin{array}{l}2,60[0,98-6,89] \\
(0,12)\end{array}$ \\
\hline Úlceras de pressão $^{d}$ & $\begin{array}{l}1,70[0,52-5,61] \\
(0,33)\end{array}$ & $\begin{array}{l}1,56[1,16-2,1] \\
(0,44)\end{array}$ & $\begin{array}{l}0,40[0,11-1,45] \\
(0,09)\end{array}$ & $\begin{array}{l}0,32[0,05-2,22] \\
(0,18)\end{array}$ \\
\hline ASA IV & $\begin{array}{l}1,91[0,61-5,99] \\
(0,24)\end{array}$ & $\begin{array}{l}1,82[1,22-2,7] \\
(0,03)\end{array}$ & $\begin{array}{l}1,15[0,57-2,32] \\
(0,48)\end{array}$ & $\begin{array}{l}2,59[1,01-6,62] \\
(0,05)\end{array}$ \\
\hline Cateterismo vesical $^{f}$ & $\begin{array}{l}1,50[0,45-5,01] \\
(0,39)\end{array}$ & $\begin{array}{l}1,69[1,2-2,4] \\
(0,11)\end{array}$ & $\begin{array}{l}0,53[0,15-1,86] \\
(0,23)\end{array}$ & $\begin{array}{l}1,54[0,56-4,2] \\
(0,32)\end{array}$ \\
\hline Permanência na UTI ${ }^{f}$ & $\begin{array}{l}2,64[0,86-8,15] \\
(0,14)\end{array}$ & $\begin{array}{l}1,60[1,17-2,18] \\
(0,28)\end{array}$ & $\begin{array}{l}0,92[0,3-2,88] \\
(0,64)\end{array}$ & $\begin{array}{l}2,19[0,79-6,06] \\
(0,18)\end{array}$ \\
\hline
\end{tabular}

${ }^{a}$ Pacientes que se moviam apenas com o auxílio de uma cadeira de rodas ou que estavam acamados antes da ocorrência da fratura

bUso de antibióticos antes da hospitalização (6 meses), ou durante a hospitalização e antes do tratamento da fratura

'Pacientes hospitalizados ou residentes em casa de repouso, hospitalização domiciliar ou sob tratamento de hemodiálise

'Presença de úlceras de pressão na admissão hospitalar ou adquiridas durante a internação e antes do tratamento da fratura

eASA, classificação do estado físico da American Society of Anesthesiologists (16)

${ }^{f}$ Cateterismo vesical ou permanência na UTI, ocorrido durante a hospitalização e antes da fratura

Mais da metade dos pacientes apresentou diagnóstico de SCoNRM positivo na admissão, enquanto que no momento do tratamento da fratura femoral $\sim 86 \%$ estavam colonizados, com o auge da incidência de colonização hospitalar ocorrendo na primeira semana. O uso de antibióticos durante a internação ou nos seis meses anteriores e o risco cirúrgico classificado como ASA IV, estavam relacionados à uma maior probabilidade de colonização por SCoNRM. Estamos considerando que essa elevação da colonização, provavelmente se deva ao aumento da necessidade de cuidado e manipulação desses pacientes.

A colonização hospitalar por SCoNRM é importante na disseminação de genes de resistência. ${ }^{3,21}$ Além disso, essas cepas apresentam especial importância nas ISC após o tratamento da FPF. 2,22,23 Considerando a alta incidência da colonização na admissão e a rápida aquisição pelos pacientes não colonizados previamente (-Fig. 1B), a adição de glicopeptídeos na profilaxia pré-operatória pode ser útil, ${ }^{6}$ já que é observada uma alta taxa de ISC por SCoNRM. Embora, essa associação possa aumentar a incidência das complicações renais $^{24}$ e causar disseminação de resistência, ela se mostra eficaz na redução da ISC. ${ }^{6}$

A prevalência de pacientes colonizados na admissão pelo KFNSE foi três vezes maior do que a prevalência de colonizados por CXNSE e duas vezes maior no momento do tratamento da fratura. Nenhum dos fatores de risco pesquisados apresentou correlação estatística com a prevalência da colonização na admissão. Em contrapartida, o risco cirúrgico classificado como ASA IV foi relacionado a um maior risco de colonização por CXNSE no momento do tratamento da fratura, assim como a incidência de colonização hospitalar. O pico da colonização por KFNSE e CXNSE ocorreu na primeira semana, assim como a colonização por SCoNRM.

Em comparação com as medidas Gram-positivas, como a higienização das mãos e outros cuidados preventivos, elas apresentam um menor impacto na prevenção da disseminação das Enterobacteriaceae resistentes, aumentando assim, a importância dos programas para racionalizar o uso dos antibióticos. ${ }^{25}$ $\mathrm{O}$ uso do cotrimoxazol na profilaxia perioperatória, com o objetivo de prevenir ISC por SARM, na cirurgia da fratura femoral levou ao aumento das infecções causadas por Gram-negativos. ${ }^{26}$

De acordo com os dados observados, a escolha da cefuroxima para profilaxia pré-operatória pode aumentar a cobertura contra as Enterobacteriaceae. Embora a colonização por CXNSE sugira o uso de outros antibióticos como profilaxia. ${ }^{26} \mathrm{O}$ risco de disseminação de novos mecanismos de resistência torna imprescindíveis estudos adicionais. ${ }^{27,28}$

Alguns fatores de risco para a colonização por bactérias resistentes mostraram relação com a redução da colonização por Enterobacteriaceae resistentes às cefalosporinas. Em destaque, está o uso prévio de antibióticos ao tratamento da fratura e a presença de úlceras de pressão. Embora não tenhamos realizado essa avaliação, acreditamos que isso se deve à disbiose intestinal e à proliferação de microrganismos não comensais. ${ }^{29}$ 


\section{Conclusão}

Destacamos aqui a incidência da colonização hospitalar e a prevalência da colonização por S. aureus, SCoNRM e Enterobacteriaceae não suscetíveis à cefazolina, estando diretamente relacionadas ao tempo de internação préoperatória. Esses dados enfatizam a importância de reduzir a internação pré-operatória em pacientes com FPF, quando não for possível deve-se implementar medidas profiláticas, como a descolonização, o isolamento e ajustes na antibioticoprofilaxia cirúrgica.

\section{Fontes de Financiamento}

Este trabalho foi financiado pelo INPRA - Instituto Nacional de Pesquisa em Resistência Antimicrobiana - Brasil, CNPq 465718/2014-0, FAPERGS 17/2551-0000514-7. Este estudo também foi parcialmente financiado pela Coordenação de Aperfeiçoamento de Pessoal de Nível Superior, Brasil (CAPES), Código Financeiro 001.

\section{Conflitos de Interesse}

Os autores não têm conflitos de interesse a declarar.

\section{Referências}

1 Humphreys H, Becker K, Dohmen PM, et al. Staphylococcus aureus and surgical site infections: benefits of screening and decolonization before surgery. J Hosp Infect 2016;94(03):295-304

2 Public Health England. Surveillance of Surgical Site Infections in NHS hospitals in England 2013/14. London Public Heal Engl2014. Available from: http://www.hpa.org.uk/Publications/InfectiousDiseases/SurgicalSiteInfectionReports/ 1311SSIreport2012to2013data/

3 Hetem DJ, Bootsma MC, Bonten MJ. Prevention of Surgical Site Infections: Decontamination With Mupirocin Based on Preoperative Screening for Staphylococcus aureus Carriers or Universal Decontamination? Clin Infect Dis 2016;62(05):631-636

4 Gillespie WJ, Walenkamp GH. Antibiotic prophylaxis for surgery for proximal femoral and other closed long bone fractures. Cochrane Database Syst Rev 2010;2010(03):CD000244

5 Bassetti M, Righi E, Astilean A, et al. Antimicrobial prophylaxis in minor and major surgery. Minerva Anestesiol 2015;81(01):76-91

6 Schweizer M, Perencevich E, McDanel J, et al. Effectiveness of a bundled intervention of decolonization and prophylaxis to decrease Gram positive surgical site infections after cardiac or orthopedic surgery: systematic review and meta-analysis. BMJ 2013;346:f2743

7 Deurenberg RH, Stobberingh EE. The evolution of Staphylococcus aureus. Infect Genet Evol 2008;8(06):747-763

8 Torbert JT, Joshi M, Moraff A, et al. Current bacterial speciation and antibiotic resistance in deep infections after operative fixation of fractures. J Orthop Trauma 2015;29(01):7-17

9 Logan LK, Weinstein RA. The epidemiology of Carbapenemresistant enterobacteriaceae: The impact and evolution of a global menace. J Infect Dis 2017;215(suppl_(Suppl 1):S28-S36

10 Marcel JP, Alfa M, Baquero F, et al. Healthcare-associated infections: think globally, act locally. Clin Microbiol Infect 2008;14 (10):895-907

11 Patel JB, Cockerill FR, Bradford PA, et al. M02-A12 Performance Standards for Antimicrobial Disk Susceptibility Tests. Wayne, PA: Clinical and Laboratory Standards Institute; 2015
12 Boye K, Bartels MD, Andersen IS, Møller JA, Westh H. A new multiplex PCR for easy screening of methicillin-resistant Staphylococcus aureus SCCmec types I-V. Clin Microbiol Infect 2007;13 (07):725-727

13 Al-Talib H, Yean CY, Al-Khateeb A, et al. A pentaplex PCR assay for the rapid detection of methicillin-resistant Staphylococcus aureus and Panton-Valentine Leucocidin. BMC Microbiol 2009;9 (01):113

14 Duran N, Temiz M, Duran GG, Eryılmaz N, Jenedi K. Relationship between the resistance genes to quaternary ammonium compounds and antibiotic resistance in staphylococci isolated from surgical site infections. Med Sci Monit 2014; 20:544-550

15 Torres K, Sampathkumar P. Predictors of methicillin-resistant Staphylococcus aureus colonization at hospital admission. Am J Infect Control 2013;41(11):1043-1047

16 Kuehnert MJ, Kruszon-Moran D, Hill HA, et al. Prevalence of Staphylococcus aureus nasal colonization in the United States, 2001-2002. J Infect Dis 2006;193(02):172-179

17 Cho SY, Chung DR. Infection Prevention Strategy in Hospitals in the Era of Community-Associated Methicillin-Resistant Staphylococcus aureus in the Asia-Pacific Region: A Review. Clin Infect Dis 2017;64(suppl_(Suppl 2):S82-S90

18 Otto M. Community-associated MRSA: what makes them special? Int J Med Microbiol 2013;303(6-7):324-330

19 Johannessen M, Sollid JE, Hanssen AM. Host- and microbe determinants that may influence the success of $S$. aureus colonization. Front Cell Infect Microbiol 2012;2:56

20 McCarthy H, Rudkin JK, Black NS, Gallagher L, O’Neill E, O'Gara JP. Methicillin resistance and the biofilm phenotype in Staphylococcus aureus. Front Cell Infect Microbiol 2015;5:1-9

21 Hurdle JG, O'Neill AJ, Mody L, Chopra I, Bradley SF. In vivo transfer of high-level mupirocin resistance from Staphylococcus epidermidis to methicillin-resistant Staphylococcus aureus associated with failure of mupirocin prophylaxis. J Antimicrob Chemother 2005;56(06):1166-1168

22 Blomfeldt R, Kasina P, Ottosson C, Enocson A, Lapidus LJ. Prosthetic joint infection following hip fracture and degenerative hip disorder: a cohort study of three thousand, eight hundred and seven consecutive hip arthroplasties with a minimum follow-up of five years. Int Orthop 2015;39(11):2091-2096

23 Ravi S, Zhu M, Luey C, Young SW. Antibiotic resistance in early periprosthetic joint infection. ANZ J Surg 2016;86(12): 1014-1018

24 Courtney PM, Melnic CM, Zimmer Z, Anari J, Lee GC. Addition of Vancomycin to Cefazolin Prophylaxis Is Associated With Acute Kidney Injury After Primary Joint Arthroplasty. Clin Orthop Relat Res 2015;473(07):2197-2203

25 Zahar J-R, Lesprit P. Management of multidrug resistant bacterial endemic. Med Mal Infect 2014;44(09):405-411

26 Gallardo-Calero I, Larrainzar-Coghen T, Rodriguez-Pardo D, et al. Increased infection risk after hip hemiarthroplasty in institutionalized patients with proximal femur fracture. Injury 2016;47 (04):872-876

27 Marchenay P, Blasco G, Navellou JC, et al. Acquisition of carbapenem-resistant Gram-negative bacilli in intensive care unit: predictors and molecular epidemiology. Med Mal Infect 2015;45(12):34-40

28 Armand-Lefèvre L, Angebault C, Barbier F, et al. Emergence of imipenem-resistant gram-negative bacilli in intestinal flora of intensive care patients. Antimicrob Agents Chemother 2013;57 (03):1488-1495

29 Khanna S, Pardi DS. Clinical implications of antibiotic impact on gastrointestinal microbiota and Clostridium difficile infection. Expert Rev Gastroenterol Hepatol 2016;10(10): $1145-1152$ 